\title{
TLR2/NFKB signalling regulates endogenous IL-6 release from marrow-derived mesenchymal stromal cells to suppress the apoptosis of PC12 cells injured by oxygen and glucose deprivation
}

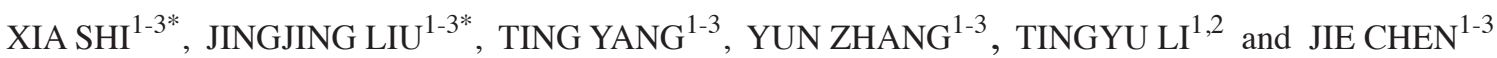 \\ ${ }^{1}$ Children's Nutrition Research Centre; ${ }^{2}$ Ministry of Education Key Laboratory of Child Development and Disorders; \\ ${ }^{3}$ Chongqing Engineering Research Centre of Stem Cell Therapy, Children's Hospital of Chongqing Medical University, \\ Chongqing 400014, P.R. China
}

Received May 10, 2015; Accepted March 29, 2016

DOI: $10.3892 / \mathrm{mmr} .2016 .5158$

\begin{abstract}
Two previous studies published by our group identified that mesenchymal stromal cells (MSCs) conferred neuroprotection in a rat model of hypoxic-ischaemic brain damage (HIBD), and that MSCs secreted abundant interleukin-6 (IL-6) when co-cultured with oxygen and glucose deprivation (OGD)-injured PC12 cells. The present study has further investigated the role of IL-6, and explored potential signalling pathways in vitro. In vitro models were established by co-culturing OGD-injured PC12 cells with MSCs. Subsequently, the expression levels of the signalling molecules, Toll-like receptor 2 (TLR2)/nuclear factor $\mathrm{\kappa B}$ $(\mathrm{NFKB})$, and IL-6 were altered separately in this in vitro model by treatment with an agonist, antagonist, siRNA or overexpression adenovirus. The expression levels of $\mathrm{B}$ cell lymphoma-associated X (Bax), TLR2, NFkB and IL-6 were detected by western blot analysis, real-time polymerase chain reaction or ELISA. The resting membrane potential (RMP) of the PC12 cells was analysed by whole-cell patch-clamp recordings. Compared with controls or the $\mathrm{PC} 12$ co-culture
\end{abstract}

Correspondence to: Professor Jie Chen, Children's Nutrition Research Centre, Children's Hospital of Chongqing Medical University, 136 Zhongshan Er Road, Chongqing 400014, P.R. China E-mail: jchen010@cqmu.edu.cn

*Contributed equally

Abbreviations: MSCs, mesenchymal stromal cells; HIBD, hypoxic-ischaemic brain damage; IL-6, interleukin-6; PC12 cells, pheochromocytoma cells; OGD, oxygen-glucose deprivation; TLR2, Toll-like receptor 2; NFאB, nuclear factor $\kappa \mathrm{B}$; Bax, B-cell lymphoma-associated X; RMP, resting membrane potential; PDTC, pyrrolidine dithiocarbamate; PGN-SA, peptidoglycan from Staphylococcus aureus

Key words: bone marrow-derived mesenchymal stromal cells, interleukin-6, Toll-like receptor 2/nuclear factor- $\kappa \mathrm{B}$ pathway, PC12 cells, oxygen and glucose deprivation group, the MSC co-cultured group induced less expression of Bax, but more IL-6 secretion. Up- or down-regulation of the TLR2/NFKB signalling pathway resulted in a corresponding increase or decrease in the IL-6 expression level in the MSCs. Co-culture with silL-6-MSCs increased the expression levels of Bax and increased the RMP in the OGD PC12 cells. In conclusion, the release of IL-6 from MSCs was regulated via the TLR2/NFkB signalling pathway. Endogenous IL-6 reduced apoptosis and protected OGD-injured PC12 cells when they were co-cultured with MSCs. The present study has reported a novel immunomodulatory effect of the microenvironment of neural damage during MSC cytotherapy.

\section{Introduction}

Cerebral hypoxic-ischaemic insult during the perinatal period leads to neonatal brain injury, including encephalopathy, motor and mental deficits, learning disabilities and epilepsy (1). Hypoxic-ischaemic brain damage (HIBD) can be caused by brain lesions and the induction of hippocampal long-term potentiation through a series of pathophysiological changes in cell metabolic dysfunction, cerebral blood flow abnormalities, excitatory amino acid neurotoxicity, and intracellular calcium overload, free radical (e.g., nitric oxide) accumulation and apoptosis $(2,3)$. The majority of drug therapies in the clinical arena are based on maintaining cell activities and delaying neuron death by preventing apoptosis to improve brain function. However, currently, no specific treatments are available for HIBD. An oxygen and glucose deprivation (OGD) in vitro model was used to simplify the complex brain interaction network in HIBD. Previous studies demonstrated that mesenchymal stromal cells (MSCs) could effectively restore learning and memory function in neonatal rats submitted to hypoxic-ischaemic insult in vivo (4-6), and these results demonstrated that transplanted MSCs exert immunomodulatory effects in the injury microenvironment, and that the MSCs secreted abundant interleukin-6 (IL-6) when co-cultured with OGD-injured PC12 cells (7). These results suggested that the high level of IL-6 expression may exert a neuroprotective role in neuronal injury. IL-6 is a pleiotropic cytokine that is able to regulate a variety of cell functions, including cell 
proliferation, cell differentiation, immune defence and the inhibition of apoptosis. Previous evidence has revealed that IL-6 acts a double-edged sword, having dual proinflammatory and anti-inflammatory effects. Several studies have revealed that the expression of IL- 6 is markedly increased when the central nervous system (CNS) is injured or is disease-afflicted, causing brain injury aggravation (8-10). However, a previous study (11) identified that astrocyte and macrophage activation were markedly decreased in the brains of IL- 6 knockout mice following brain injury. This decrease in immunological reactivity suggested that IL-6 may have a neuroprotective role in brain injury. Based on these findings, the present study aimed to identify how MSCs secrete IL-6, and the physiological role of IL-6 in OGD-injured PC12 cells.

\section{Materials and methods}

Animals. Ten Wistar rats (age, 21 days), including 5 female and 5 male rats, were purchased from the Animal Experiment Centre of Daping Hospital affiliated with the Third Military Medical University (Chongqing, China). The animals were housed under a $12 \mathrm{~h}$ light/dark cycle with food and water freely available [SYXK (Yu) 2012-0015]. The experimental animal procedures were approved by the Ethics Committee of Chongqing Medical University (Chongqing, China).

Isolation and culture of MSCs. MSCs were isolated from the bone marrow of 21-day-old Wistar rats. The bone marrow was washed repeatedly with Dulbecco's modified Eagle's medium (DMEM/F12; Gibco, Thermo Fisher Scientific, Inc., Waltham, MA, USA) in a laminar flow cabinet until the bone had become bleached and the bone marrow was flushed out. Subsequently, the fluid containing bone marrow was centrifuged at $10,000 \mathrm{x} \mathrm{g}$ at room temperature for $3 \mathrm{~min}$, and the supernatant was discarded. Then, the bone marrow cells were scattered and plated in a $60 \mathrm{~mm}$ Petri dish. The DMEM/F12 culture medium with $10 \%$ foetal bovine serum (FBS; Gibco, Thermo Fisher Scientific, Inc.) was changed every $24 \mathrm{~h}$ for the first three days, and subsequently the medium was exchanged every other day for three additional days. When the MSCs reached $70-80 \%$ confluence, the cells were digested with TrypLE (Gibco; Thermo Fisher Scientific, Inc.) and passaged at a ratio of $1: 2$ or $1: 3$.

Culturing PC12 cells. PC12 cells were obtained from the Cell Bank of Shanghai Institute of Cell Biology, Chinese Academy of Science (Shanghai, China). The cells were cultured in DMEM containing $10 \%$ horse serum, $5 \%$ FBS, $100 \mathrm{U} / \mathrm{ml}$ penicillin and $100 \mu \mathrm{g} / \mathrm{ml}$ streptomycin (all reagents purchased from Gibco; Thermo Fisher Scientific, Inc.) at $37^{\circ} \mathrm{C}$ in a $5 \% \mathrm{CO}_{2}$ incubator. When the PC12 cells reached $90 \%$ confluence, they were digested with TrypLE (Gibco; Thermo Fisher Scientific, Inc.) and passaged at a ratio of 1:2 every three to four days.

InvitroOGDmodelconstructionandco-cultureconfigurations. The PC12 cells were seeded and grown to $\sim 90 \%$ confluence in a six-well plate. To establish the OGD model, the PC12 cell culture medium was changed to Earle's balanced salt solution (EBSS; GE Healthcare Life Sciences, Logan, Utah, USA) after the cells had been washed twice with D-Hank's solution
(GE Healthcare Life Sciences). Subsequently, the cells were placed in an incubator (Thermo Forma 3111; Thermo Fisher Scientific, Inc.) with $5 \% \mathrm{O}_{2}$ and $95 \% \mathrm{~N}_{2}$ at $37^{\circ} \mathrm{C}$ for $4 \mathrm{~h}$, after which the EBSS medium was replaced with normal cell medium. The OGD-injured PC12 cells were co-cultured with MSCs $\left(2 \times 10^{6}\right.$; called the MSC co-culture group) or with normal PC12 cells ( $2 \times 10^{6}$; called the PC12 co-culture group) for a further $24 \mathrm{~h}$. OGD-injured PC12 cells without co-culture served as controls. The co-culture methods were performed as previously described (12), and the PC12 cell co-culture group without injury was used as a co-culture negative control.

MSC treatments. To up-regulate Toll-like receptor 2 (TLR2) or down-regulate $\mathrm{NF} \kappa \mathrm{B}$, the MSCs were cultured in the presence of the TLR2 agonist, peptidoglycan from Staphylococcus aureus (PGN-SA; $8 \mu \mathrm{g} / \mathrm{ml}$; InvivoGen, Hong Kong, China) or the NFאB inhibitor, pyrrolidine dithiocarbamate (PDTC; $10 \mu \mathrm{g} / \mathrm{ml}$; Sigma-Aldrich, St. Louis, MO, USA) for $24 \mathrm{~h}$. Subsequently, the MSCs were treated with a TLR2- or IL-6-targeting small interfering (si)RNA, siTLR2 and siIL-6, or recombinant Ad-IL-6 adenovirus to knock down the expression of TLR 2 or to regulate the expression level of IL-6, respectively, according to our previous studies $(13,14)$. MSCs treated with red fluorescent protein (RFG) served as the control. Stable siIL-6-MSCs were used to investigate the biological function of IL-6, as reported previously (15). Green fluorescent protein (GFP)-labelled MSCs were used as a control.

RNA isolation and real-time quantitative polymerase chain reaction ( $R T-q P C R)$ analysis. Total RNA was isolated using an RNA extraction kit (BioTek Corp., Beijing, China) and reverse-transcribed into cDNA using a PrimeScript ${ }^{\circledR}$ RT Reagent kit (DRR037A; Takara Bio, Inc., Shiga, Japan) and a Bio-Rad My Cycler (Bio-Rad Laboratories, Inc., Hercules, CA, USA), according to the manufacturer's protocol. The single-stranded cDNA was diluted 10 -fold, and used as the template. qPCR was performed on a StepOne ${ }^{\mathrm{TM}}$ v2.1 Real-Time PCR instrument (Applied Biosystems; Redwood City, CA, USA) using Real Master mix [SYBR ${ }^{\circledR}$ Green; Tiangen Biotech (Beijing) Co., Ltd, Beijing, China]. The thermocycling conditions were as follows: Initial denaturation at $95^{\circ} \mathrm{C}$ for $30 \mathrm{sec}$ and denaturation at $95^{\circ} \mathrm{C}$ for $5 \mathrm{sec}$, followed by 40 cycles at $60^{\circ} \mathrm{C}$ for $30 \mathrm{sec}$. Melting curve analysis and gel electrophoresis were performed to ensure that a single PCR product was amplified in each reaction. The PCR primers were designed using Primer Premier 5.0 software (Premier Biosoft International, Palo Alto, CA, USA). The ratio of the relative quantity of the target gene to glyceraldehyde-3-phosphate dehydrogenase (GAPDH), as a reference gene, was calculated using the $2^{-\Delta \Delta \mathrm{Cq}}$ method (16). The primer sequences for GAPDH, TLR2, NFאB and IL- 6 are shown in Table I.

Western blot analysis. The cells were lysed in radioimmunoprecipitation assay buffer containing phenylmethanesulfonyl fluoride (Bioteke Co., Ltd., Taiwan, China). The cell lysates were collected, and the protein concentration was determined using a bicinchoninic acid protein concentration determination kit (Bioteke Co., Ltd., Beijing, China). The cell lysates were purified by $10 \%$ sodium dodecyl sulphate-polyacrylamide gel 
Table I. Primer sequences for polymerase chain reaction.

Gene

Primer sequence

$\begin{array}{ll}\text { GAPDH } & \text { F: 5'-CCTGGAGAAACCTGCCAAG-3' } \\ & \text { R: 5'-CACAGGAGACAACCTGGTCC-3' } \\ \text { TLR2 } & \text { F: 5'-TCTCGGCAACTATGAGTCCC-3' } \\ & \text { R: 5'-ATCGGTGAGATCTGCATTCC-3' } \\ \text { NFאB } & \text { F: 5'-AGGACTGCCGGGATGGCTTCTAT-3' } \\ & \text { R: 5'-GGTCTGGATGCGCTGGCTAATGG-3' } \\ \text { IL-6 } & \text { F: 5'-ACAGCCACTGCCTTCCCTAC-3' } \\ & \text { R: 5'-TTGCCATTGCACAACTCTTTTC-3' }\end{array}$

F, forward; R, reverse; GAPDH, glyceraldehyde-3-phosphate dehydrogenase; TLR2, Toll-like receptor 2 ; $\mathrm{NF \kappa B}$, nuclear factor $\kappa \mathrm{B}$; IL-6, interleukin-6.

electrophoresis (Beyotime Institute of Biotechnology, Beijing, China), with 30-40 $\mu$ g total protein loaded per lane. After the proteins were electrophoresed, they were transferred to polyvinylidene fluoride membranes (Millipore Corp., Billerica, MA, USA). Following blocking of the membranes with $5 \%$ bovine serum antigen in Tris-buffered saline/Tween-20 (TBST) buffer at room temperature for $1 \mathrm{~h}$, they were probed overnight with primary antibodies, including mouse monoclonal anti-B-cell lymphoma-associated X (anti-Bax; cat. no. sc-7480; 1:1,000; Santa Cruz Biotechnology, Inc., CA, USA), rabbit polyclonal anti-TLR2 (cat. no. SAB2102440; 1:1,000; Sigma-Aldrich), rabbit polyclonal anti-NFKB (cat. no. ab12146; 1:500 to 1:1,000; Abcam, Cambridge, UK), mouse monoclonal anti-IL-6 (cat. no. MAB5061; 1:500; R\&D Systems China Co., Ltd., Shanghai, China), and mouse monoclonal $\beta$-actin (cat. no. sc-47778; 1:100 to 1:1,000; Santa Cruz Biotechnology, Inc.) at $4^{\circ} \mathrm{C}$, and subsequently incubated with the appropriate secondary antibody conjugated with horseradish peroxidase (HRP; Santa Cruz Biotechnology, Inc.) at room temperature for $1 \mathrm{~h}$. The proteins were detected using an enhanced chemiluminescent (ECL) substrate kit containing chemiluminescent HRP substrate (Millipore Corp.), and protein levels were recorded using an ECL Imaging System (G:BOX; Syngene UK, Cambridge, UK).

Enzyme-linked immunosorbent assay (ELISA). The levels of IL-6 cytokine released into the culture media of the different treatment groups were measured with an ELISA kit (Beijing 4A Biotech Co., Ltd., Beijing, China), according to the manufacturer's protocol. Background values were also analysed as a control. The optical density absorbance was measured at a wavelength of $450 \mathrm{~nm}$. The values were calculated based on a constructed standard curve, and the assay was performed in triplicate.

Whole-cell patch-clamp recordings. The resting membrane potential (RMP) of cells was measured with a whole-cell patch clamp, as previously reported (4). Briefly, $30 \mathrm{~mm}$ glass coverslips containing PC12 cells, following co-culture with GFP-labelled MSCs or silL-6-MSCs, were placed in an acrylic chamber under a TE-2000U fluorescence inverted microscope (Nikon, Tokyo, Japan). The membrane potential was amplified using a Multiclamp 700B amplifier, and a Digidata 1322 interface (Axon Instruments, Foster City, CA, USA) was used for acquisition and off-line analysis. Between seven and ten intact cells in each group were selected to record the RMP, and all the recordings were performed at room temperature and completed within $1 \mathrm{~h}$ following the removal of the cells from the incubator.

Statistical analysis. All the data are expressed as the mean \pm standard error of the mean. Significant differences between samples were examined by repeated measurements of analysis of variance (ANOVA) with one-way ANOVA, followed by Duncan's multiple-range test. $\mathrm{P}<0.05$ was considered to indicate a statistically significant value.

\section{Results}

MSCs down-regulate the expression level of the apoptosis factor, Bax, and up-regulate the expression level of IL-6 in OGD-injured PC12 cells. To evaluate the effects of MSCs on the OGD-injured PC12 cells, an in vitro co-culture system of MSCs and OGD-injured PC12 cells was established. Bax protein expression in the OGD-injured PC12 cells, which were co-cultured with the same quantity of uninjured PC12 cells or MSCs, was analysed by western blot analysis. As shown in Fig. 1A, the protein expression level of Bax in the MSC co-culture group was the lowest, that in the uninjured PC12 cell co-culture group was at an intermediate level, and that in the OGD-injured PC12 cell group was the highest. These data demonstrated that co-culture with MSCs was able to promote anti-apoptotic effects on the OGD-injured PC12 cells. The supernatants of the three above-mentioned groups were collected to assess IL-6 expression by ELISA; as shown in Fig. 1B, IL-6 secretion in the MSC co-culture group was significantly higher compared with that in the uninjured $\mathrm{PC} 12$ cell co-culture group $(\mathrm{P}<0.001)$, whereas no significant difference in IL- 6 secretion was observed between the uninjured $\mathrm{PC} 12$ cell co-culture group and the OGD-injured PC12 cell group. These data demonstrated that IL-6 was secreted primarily by MSCs, which may regulate the anti-apoptotic effect on the OGD-injured PC12 cells.

Protein expression levels of TLR2, NFKB and IL-6 are altered following PGN-SA and siTLR2 treatment of MSCs. To investigate whether IL- 6 expression was regulated via the TLR2/NFKB signalling pathway, the MSCs were treated with PGN-SA or siTLR2. The changes in the expression levels of TLR2, NFKB and IL- 6 were detected by RT-qPCR and western blot analysis. As shown in Fig. 2A, when compared with the control group, the mRNA expression levels of TLR2 $(\mathrm{P}<0.001), \mathrm{NF} \kappa \mathrm{B}(\mathrm{P}<0.01)$ and IL-6 $(\mathrm{P}<0.05)$ were significantly increased following PGN-SA treatment. The changes in TLR2, NFKB and IL-6 protein expression detected by western blot analysis were identical with those changes in mRNA expression (Fig. 2B), suggesting that treatment with $8 \mu \mathrm{g} / \mathrm{ml}$ PGN-SA not only increased the expression level of TLR2 efficiently, but also up-regulated the expression of NFKB and IL-6. Subsequently, the MSCs were infected with siTLR2 adenovirus; compared with the RFP group, the mRNA and protein expression levels of TLR2 $(\mathrm{P}<0.01), \mathrm{NF} \kappa \mathrm{B}$ $(\mathrm{P}<0.05)$ and IL-6 $(\mathrm{P}<0.001)$ were significantly decreased in 
A

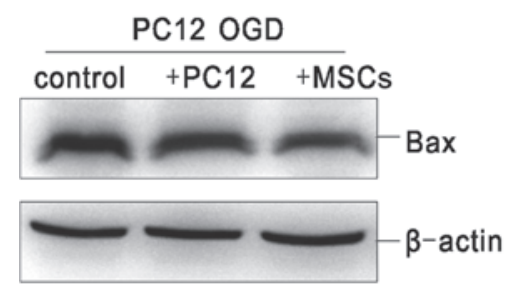

B

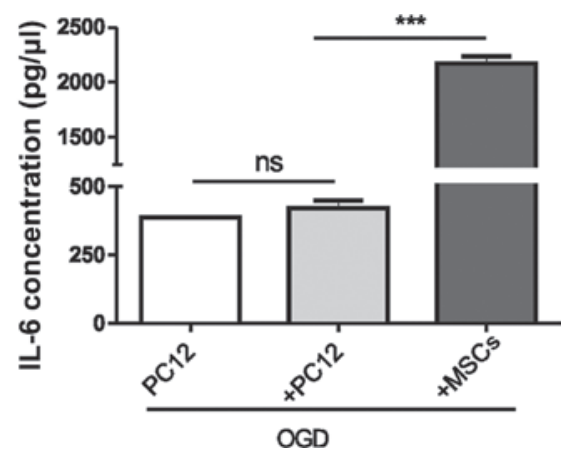

Figure 1. OGD-injured PC12 cells show an increased secretion of IL-6 and decreased protein levels of Bax when co-cultured with MSCs. (A) Detection of Bax protein levels in OGD-injured PC12 cells cultured alone and in OGD-injured PC12 cells co-cultured with uninjured PC12 cells or MSCs. A western blot representative of three independent experiments is shown. (B) Concentration of IL-6 in the supernatants of OGD-injured PC12 cells cultured alone, and of OGD-injured PC12 cells co-cultured with uninjured PC12 cells or MSCs, as detected by ELISA. Values are expressed as the mean \pm standard error of the mean $(\mathrm{n}=3){ }^{* * * *} \mathrm{P}<0.001$, vs. the uninjured PC12 co-culture group. MSCs, mesenchymal stromal cells; Bax, Bcl-2-associated X; IL-6, interleukin 6; OGD, oxygen-glucose deprivation; ELISA, enzyme-linked immunosorbent assay; ns, not significant.

the siTLR2 group (Fig. 2C and D). The above results demonstrated that the down-regulation of NFkB and IL-6 expression levels may be associated with reduced levels of TLR2, and further suggested that MSCs may regulate the secretion of IL-6 via the TLR2/NFKB/p65 signalling pathway.

The levels of $N F \kappa B$ and IL- 6 protein expression are down-regulated by the $N F \kappa B$ inhibitor, PDTC. To evaluate whether IL-6 is released from the MSCs via the TLR2/NFKB

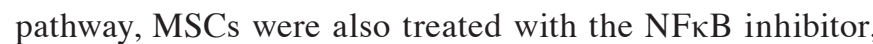
PDTC. Following treatment of the MSCs with $10 \mu \mathrm{g} / \mathrm{ml}$ PDTC for $48 \mathrm{~h}$, the protein levels of NFKB, IL-6 and TLR2 were assessed by western blot analysis, and the quantification of the results is shown in Fig. 3. Significant decreases in the expression levels of NFKB $(\mathrm{P}<0.001)$ and IL-6 $(\mathrm{P}<0.005)$ were observed following PDTC treatment compared with the control group. However, the expression levels of TLR2 were not significantly different between the PDTC group and the control group (Fig. 3A and B). This finding suggested that the inhibition of $\mathrm{NF \kappa B}$ expression was able to decrease IL-6 expression, although it did not affect TLR2 expression.

siIL-6 and Ad-IL-6 alter IL-6 expression, but do not affect protein expression in the TLR2/NF $\mathrm{K}$ pathway. To investigate whether IL-6 may regulate via a feedback process the TLR2/NFאB signalling pathway, MSCs were treated with Ad-IL-6 or siIL-6, and the expression levels of IL-6, TLR2 and $\mathrm{NF} \kappa \mathrm{B}$ were analysed by RT-qPCR and western blot analysis. The data in Fig. 4A and B demonstrate that IL-6 expression was markedly increased in the MSCs treated with Ad-IL-6 $(\mathrm{P}<0.001)$ compared with that of the RFP group, whereas the expression of TLR2 and NFאB did not change. Subsequently, the MSCs were infected with silL-6, and as expected, the IL-6 expression level correspondingly decreased $(\mathrm{P}<0.001)$; however, silL-6 infection did not reduce the expression levels of TLR2 or NFאB (Fig. 4C and D). These findings suggested that siIL-6 and Ad-IL-6 were able to change the expression level of IL-6, but did not affect the expression levels of TLR2 or NFKB in the MSCs.

silL-6-MSCs induce Bax expression and affect the RMP in the OGD-injured PC12 cells. To evaluate the biological function of IL-6 secreted from MSCs co-cultured with the OGD-injured
PC12 cells, the MSC cell line was screened for stable silL-6 expression (15). The protein expression level of Bax was markedly increased in the OGD-injured PC12 cells co-cultured with the siIL-6-MSCs compared with the GFP-MSC group (Fig. 5A). The whole-cell patch-clamp recordings revealed that the RMP of the siIL-6-MSC group experienced a greater increase compared with that of the GFP-MSC group ( $\mathrm{P}<0.05$; Fig. 5B). These findings indicated that endogenous IL- 6 from MSCs improved the restorative function of the OGD-injured PC12 cells.

\section{Discussion}

A previous study (11) has demonstrated that transplanted MSCs are not only able to ameliorate newly sustained brain damage, but are also able to improve the long-term prognosis in vivo. Transplanted MSCs have neuroprotective effects on the treatment of CNS injury, including traumatic brain injury and stroke, as well as on animal models of spinal cord injury. Previous studies $(4,5,17)$ by our group have shown that MSCs can be induced and differentiated into neurons to promote the recovery of nerve function in HIBD rats. A further study (7) indicated that MSCs may secrete large quantities of IL-6 and IL-10, and that the levels of these cytokines may change the injury microenvironment and reduce $\mathrm{H}_{2} \mathrm{O}_{2}$-induced apoptosis. These results suggested that transplanted MSCs may have direct or indirect immunomodulatory effects on the injury microenvironment. In the present study of MSCs co-cultured with OGD-injured PC12 cells, it was demonstrated that the expression level of Bax in the MSC co-cultured group was significantly lower compared with that in the PC12 co-cultured and control groups, suggesting that MSCs have neuroprotective effects on OGD-injured PC12 cells.

An ELISA assay of the supernatants revealed that IL-6 secretion in the MSC co-culture group was significantly higher compared with that in the $\mathrm{PC} 12$ cell co-culture group, indicating that IL- 6 be involved in the neuroprotective effects of MSCs and in microenvironment regulation. IL- 6 is a secreted protein that consists of 184 amino acids, which is widely present in the human body, specifically in T cells, B cells, glial cells, fibroblasts, epithelial cells and certain tumour cells $(18,19)$. IL-6 is able to generate an immune response, and has a role in macrophage and astrocyte activation during CNS injury. Under normal physiological condi- 
A

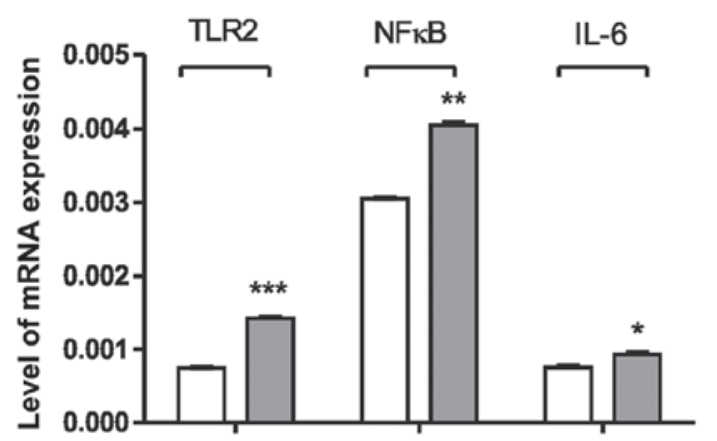

C

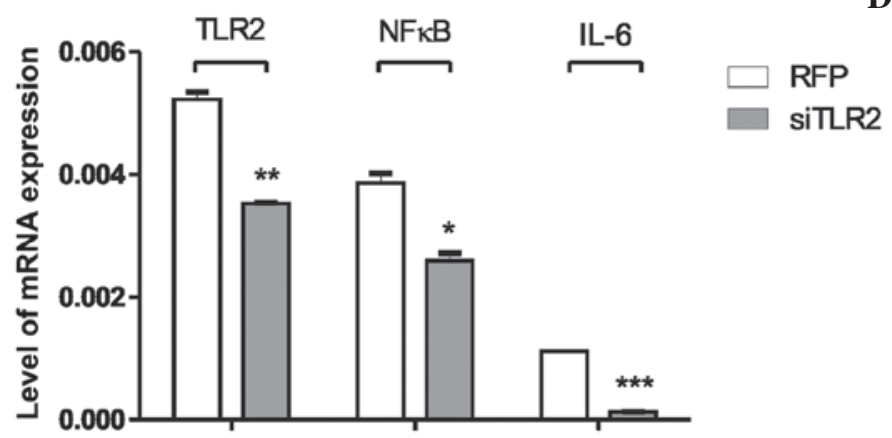

B

control

PGN-SA

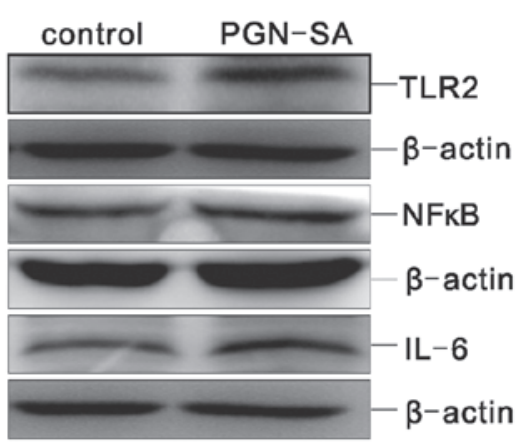

D

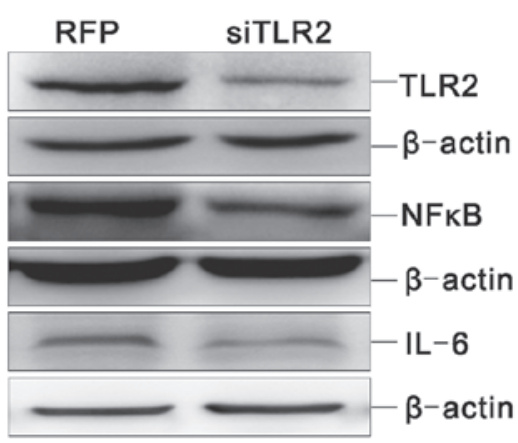

Figure 2. PGN-SA and siTLR2 caused changes in the TLR2/NFאB pathway and IL-6, respectively, in MSCs. (A) RT-qPCR was used to assess mRNA levels of TLR2, NFkB and IL-6 expression in MSCs after being treated with PGN-SA. The values are expressed as the mean \pm standard error of the mean ("P<0.05, ${ }^{* *} \mathrm{P}<0.01,{ }^{* * *} \mathrm{P}<0.001$ vs. the respective control group cultured without PGN-SA). (B) Western blot analysis of the protein levels of TLR2, NFאB and IL-6 in MSCs following treatment with PGN-SA. (C) Real-time PCR was used to assess mRNA levels of TLR2, NFkB and IL-6 expression in MSCs after being treated with siTLR2. The values are expressed as the mean \pm standard error of the mean $\left({ }^{*} \mathrm{P}<0.05,{ }^{* *} \mathrm{P}<0.01,{ }^{* * *} \mathrm{P}<0.001\right.$ vs. the respective control RFP group). (D) Western blot analysis of the protein levels of TLR2, NFאB and IL-6 in MSCs following treatment with siTLR2. For the western blot analyses (B and D), the data were obtained from at least three independent experiments, analysed and normalised against $\beta$-actin. MSCs, mesenchymal stromal cells; RT-qPCR, real-time quantitative polymerase chain reaction; TLR2, Toll-like receptor 2; IL-6, interleukin-6; NFkB, nuclear factor $\kappa B$; PGN-SA, peptidoglycan from Staphylococcus aureus; siTLR2, Toll-like receptor 2 small interfering RNA; RFP, red fluorescent protein.

A

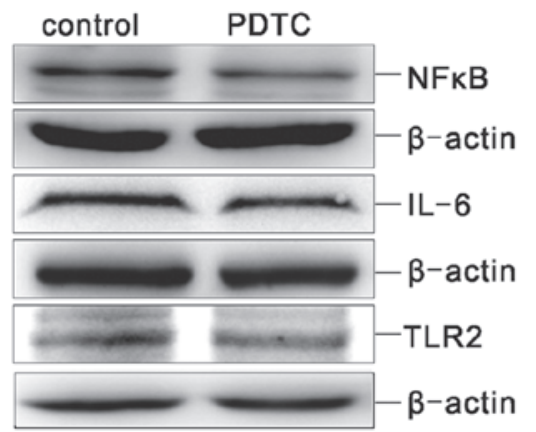

B

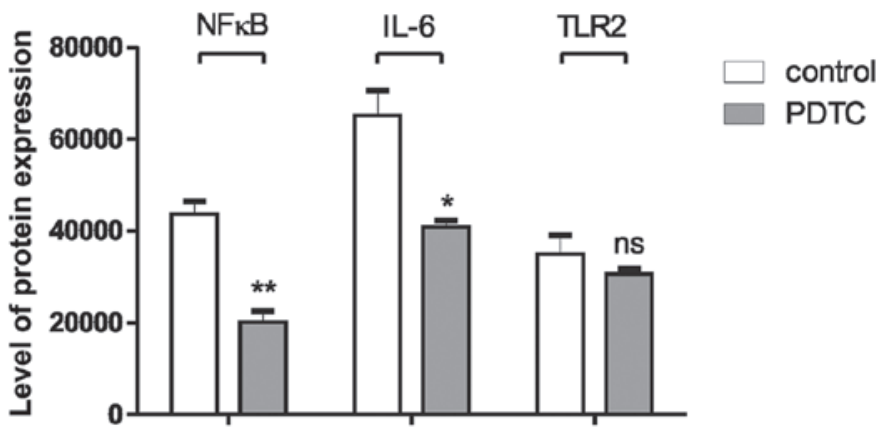

Figure 3. Protein expression levels of NFKB, IL-6 and TLR2 were assessed in MSCs following treatment with the NFkB inhibitor, PDTC. (A) Western blot analysis of NFאB, IL-6 and TLR2 protein expression in MSCs following treatment with $10 \mu \mathrm{g} / \mathrm{ml}$ PDTC. (B) Quantification (grey intensity analysis) of the western blot results. The data were obtained from at least three independent experiments, analysed and normalised against $\beta$-actin. The values are expressed as the mean \pm standard error of the mean $\left(" \mathrm{P}<0.05,{ }^{* *} \mathrm{P}<0.01\right.$ vs. the respective control group). MSCs, mesenchymal stromal cells; TLR2, Toll-like receptor 2; $\mathrm{NF \kappa B}$, nuclear factor $\kappa \mathrm{B}$; IL-6, interleukin-6; PDTC, pyrrolidine dithiocarbamate.

tions, the expression level of IL-6 in the brain is extremely low. However, when the CNS is injured or becomes disease-afflicted, the level of IL-6 is significantly increased to induce the release of tumour necrosis factor- $\alpha$ (TNF- $\alpha$ ) and IL- $1 \beta$, leading brain injury aggravation. However, Erta et al (11), in studying IL-6 knockout mice following brain injury, demonstrated that astrocyte and macrophage activation markedly decreased in the injured brain, and that the immunological reaction was reduced, suggesting that IL-6 is a key regulatory cytokine during brain injury. By contrast, another study (20) revealed that a high expression of IL-6 could reduce brain ischaemic injury, demonstrating that IL-6 also exerted neuroprotective effects. These results demonstrated that IL-6 exerts dual proinflammatory and anti-inflammatory effects. To evaluate the biological function of IL-6 secreted from MSCs co-cultured with OGD-injured PC12 cells, an MSC cell line was screened in the present study 
A

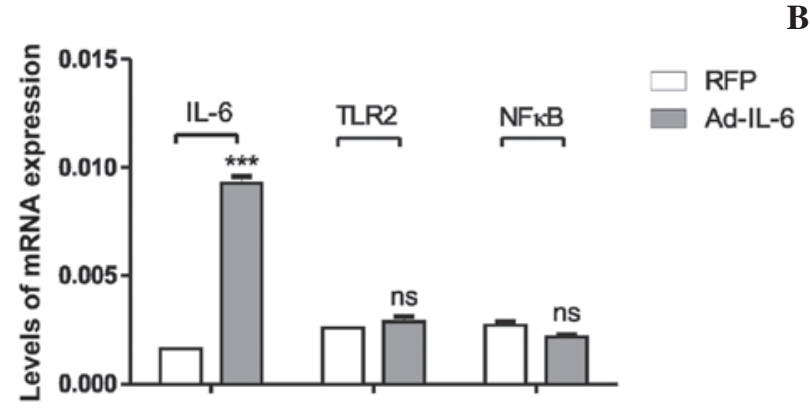

C

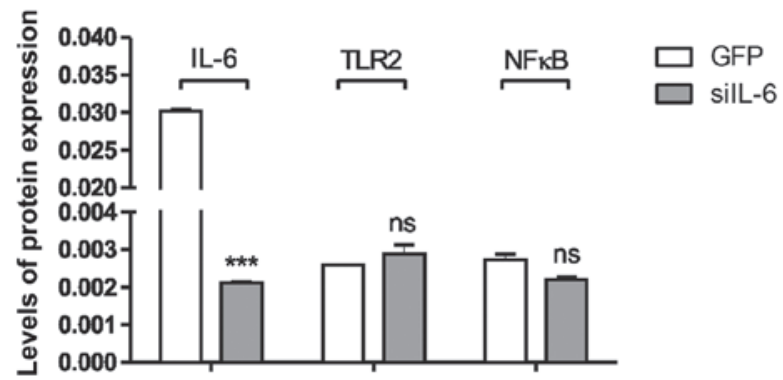

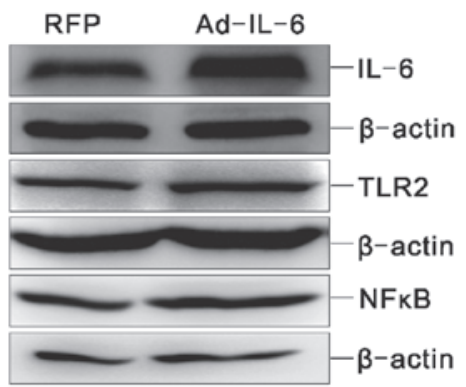

D

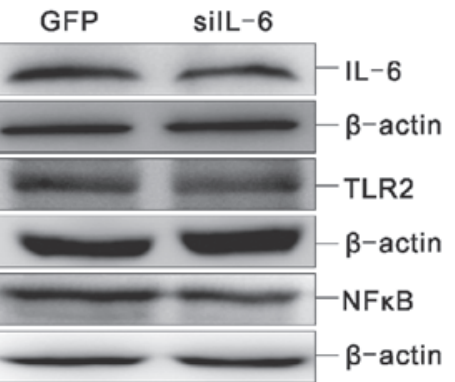

Figure 4. Determination of the changes in the TLR2/NFkB /IL-6 pathway in MSCs following treatment with Ad-IL-6 or siIL-6. (A) RT-qPCR analysis of the mRNA expression levels of IL-6, TLR2 and NFkB in MSCs following treatment with Ad-IL-6. The values are expressed as the mean \pm standard error of the mean. ${ }^{* * *} \mathrm{P}<0.001$ vs. the RFP group. (B) Western blot analysis of IL-6, TLR2 and NFKB protein levels in MSCs following treatment with Ad-IL-6. (C) RT-qPCR analysis of the mRNA expression levels of IL-6, TLR2 and NFкB in MSCs following treatment with siIL-6. The values are expressed as the mean \pm standard error of the mean. ${ }^{* * *} \mathrm{P}<0.001$ vs. the RFP group. (D) Western blot analysis of IL-6, TLR2 and NFkB protein levels in MSCs following treatment with siIL-6. For the western blot analyses (B and D), the data were obtained from at least three independent experiments, analysed and normalised against $\beta$-actin. MSCs, mesenchymal stromal cells; NFkB, nuclear factor кB; IL-6, interleukin-6; Ad-IL-6, IL-6 adenovirus; RFP, red fluorescent protein; silL-6, IL-6 small interfering RNA; GFP, green fluorescent protein; TLR2, Toll-like receptor 2.

A

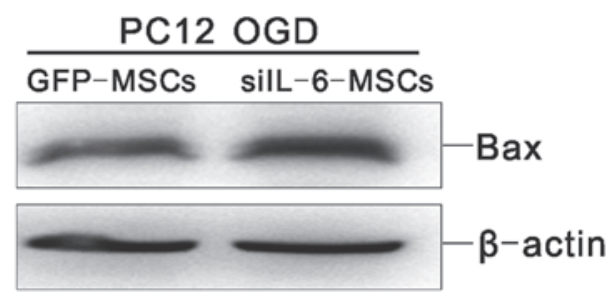

B

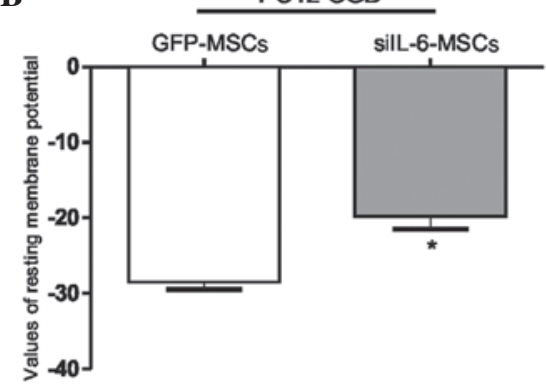

Figure 5. Effect of siIL-6-MSCs on the (A) expression level of Bax and (B) RMP in OGD-injured PC12 cells. (A) Changes in Bax protein expression in OGD-injured PC12 cells co-cultured with GFP-MSCs or silL-6-MSCs. (B) Changes in the RMP of OGD-injured PC12 cells following co-culture with GFP-MSCs and silL-6-MSCs, as determined by whole-cell patch-clamp recordings. The values are presented as the mean \pm standard error of the mean for data from 6-8 samples ( $\mathrm{P}<0.05$ vs. the GFP-MSC co-culture group). GFP, green fluorescent protein; MSC, mesenchymal stromal cells; RMP, resting membrane potential; siIL-6, interleukin-6 small interfering RNA; Bax, B-cell lymphoma-associated X; OGD, oxygen and glucose deprivation.

with stable silL-6 expression. When the expression level of IL-6 decreased, the expression level of Bax increased and the RMP was induced. Bax is an apoptotic factor, and the increase in Bax expression levels indicated that apoptosis of the OGD-injured PC12 cells was aggravated. The RMP is the membrane potential of a nerve cell in an unstimulated state, and is known as the resting potential or transmembrane resting potential. The RMP threshold of neurons represents their viability and transmitting function. The whole-cell patch-clamp recordings revealed that the RMP threshold of the OGD-injured PC12 cells following co-culture with siIL-6-MSCs experienced a greater increase compared with that of the GFP-MSC co-culture group. These findings indicated that the decrease in the expression levels of
IL-6 in the MSCs reduced the capability of the OGD-injured PC12 cells to recover, thereby suggesting that endogenous IL-6 secreted from MSCs improved the functional restoration of OGD-injured PC12 cells.

The present study suggested that endogenous IL- 6 secreted from MSCs had neuroprotective effects; however, the mechanism by which the MSCs secreted IL-6, and the signalling pathway(s) that are involved, have yet to be fully elucidated. In this preliminary study, the expression levels of TLR2 and IL-6 were shown to be increased when the PC12 cells were subjected to OGD injury. In the CNS, TLRs are not only key components of the innate immune system, but they also are associated with nerve degeneration and tissue damage $(21,22)$. TLR2 is a member of 
the TLR family that is highly expressed in the microglia of the CNS. NFאB is activated by TLR2 via the myeloid differentiation primary response gene 88 (MyD88) pathway in the presence of interleukin-1 receptor-associated kinase (IRAK)-1 and IRAK-4, inducing the production of cytokines and chemical factors, including IL-1, IL-6 and TNF (23-25). In the current study, to investigate whether endogenous IL-6 secretion from MSCs was also regulated via the TLR2/NFkB signalling pathway, the MSCs were treated with the TLR2 agonist, PGN-SA. Compared with the control group, the expression levels of TLR2, NFkB and IL-6 increased following PGN-SA treatment. After the MSCs had been treated with siTLR2 adenovirus, the expression levels of TLR2, NFKB and IL-6 markedly decreased. These results demonstrated that TLR2 expression in MSCs directly

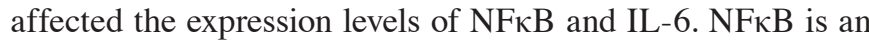
important factor in the TLR2 signalling pathway, and exerts a key role in the induction of trans-shipment in the innate immune system (26-28). The NFאB inhibitor, PDTC, was also used in the present study to treat MSCs. Following treatment of the MSCs with $10 \mu \mathrm{g} / \mathrm{ml}$ PDTC for $48 \mathrm{~h}$, a significant decrease in the expression level of IL-6 in the MSCs was observed. However, the expression level of TLR2 was not significantly different compared with untreated MSCs. These data further demonstrated that endogenous IL-6 secreted from MSCs was regulated via the TLR2/NFKB signalling pathway.

At this stage, it had not been established whether IL-6 feedback regulated the expression levels of TLR2 and NFкB. To investigate this hypothesis, MSCs were treated with Ad-IL-6 adenovirus and siIL-6, and Ad-IL-6 and siIL-6 were revealed to change the expression level of IL-6, but not affect the expression levels of TLR2 or NFKB in the MSCs. These data suggested that endogenous IL-6 secreted from MSCs was regulated by the TLR2/NFkB signalling pathway, although IL-6 did not regulate the expression levels of TLR2 and NFKB via a feedback mechanism. Therefore, further studies should focus on the biological effects of endogenous IL-6 from MSCs on the process of HIBD treatment.

In conclusion, the release of IL-6 from MSCs was regulated via the TLR2/NFאB signalling pathway. Endogenous IL-6 secreted from MSCs was able to reduce the levels of apoptosis and to improve the functional restoration of OGD-injured PC12 cells following co-culture with MSCs. These findings represent a novel immunomodulatory effect of the neural injury microenvironment during MSC cytotherapy.

\section{Acknowledgements}

This study was supported by grants from the National Natural Science Foundation of China (nos. 81271385 and 81300522) and the Stem Cell Therapy for Special Study of Children's Hospital of Chongqing Medical University (no. SCT-201203).

\section{References}

1. Calvert JW and Zhang JH: Pathophysiology of an hypoxic-ischemic insult during the perinatal period. Neurol Res 27: 246-260, 2005.

2. Khot S and Tirschwell DL: Long-term neurological complications after hypoxic-ischemic encephalopathy. Semin Neurol 26: 422-431, 2006.

3. Kristián T: Metabolic stages, mitochondria and calcium in hypoxic/ischemic brain damage. Cell Calcium 36: 221-233, 2004
4. Bi Y, Gong M, Zhang X, Zhang X, Jiang W, Zhang Y, Chen J, Liu Y, $\mathrm{He}$ TC and Li T: Pre-activation of retinoid signaling facilitates neuronal differentiation of mesenchymal stem cells. Dev Growth Differ 52: 419-431, 2010

5. Liu Y, Zhang X, Dai Y, Shu C, Qu P, Liu YX, Yang L and Li TY: Effects of bone marrow mesenchymal stem cells on learning and memory functional recovery in neonatal rats with hypoxic-ischemic brain damage. Zhonghua Er Ke Za Zhi 46: 648-653, 2008 (In Chinese).

6. Phinney DG and Isakova I: Plasticity and therapeutic potential of mesenchymal stem cells in the nervous system. Curr Pharm Des 11: 1255-1265, 2005.

7. Zhang Y, Yang Y, Bi Y, Gong M, Jiang W, Wei X, Li T and Chen J: Mesenchymal stromal cell neuroprotection of hydrogen peroxide-challenged pheochromocytoma cells through reducing apoptosis and releasing cytokines. Cytotherapy 14: 954-966, 2012.

8. Winter CD, Pringle AK, Clough GF and Church MK: Raised parenchymal interleukin-6 levels correlate with improved outcome after traumatic brain injury. Brain 127: 315-320, 2004

9. Hüll M, Strauss S, Berger M, Volk B and Bauer J: The participation of interleukin-6, a stress-inducible cytokine, in the pathogenesis of Alzheimer's disease. Behav Brain Res 78: 37-41, 1996.

10. Qiu Z, Sweeney DD, Netzeband JG and Gruol DL: Chronic interleukin-6 alters NMDA receptor-mediated membrane responses and enhances neurotoxicity in developing CNS neurons. J Neurosci 18: 10445-10456, 1998

11. Erta M, Quintana A and Hidalgo J: Interleukin-6, a major cytokine in the central nervous system. Int J Biol Sci 8: 1254-1266, 2012.

12. Chen J, Ng CP, Rowlands DK, Xu PH, Gao JY, Chung YW and Chan HC: Interaction between enteric epithelial cells and Peyer's patch lymphocytes in response to Shigella lipopolysaccharide: Effect on nitric oxide and IL-6 release. World J Gastroenterol 12: 3895-3900, 2006.

13. Zhang Y, Gong M, Bi Y, Jiang W, Yu Q, Li TY and Chen J: Construction and identification of recombinant adenovirus vector containing siRNA of rat TLR2 gene. Xi Bao Yu Fen Zi Mian Yi Xue Za Zhi 28: 144-147, 2012 (In Chinese).

14. Liu JJ, Zhang YJ, Bi Y, Gong M, Li T-y and Chen J: Construction and preliminary identification of rat interleukin- 6 recombinant adenovirus. Immunol J 30: 151-155, 2014.

15. He M-l, Liu J-j, Gu Y, L T-y and Chen J: Effects of inhibiting secretion of mesenchymal stem cells originated interleukin- 6 on oxygen glucose deprivation injured PC12 cells. J Shanghai Jiaotong Uni (Sci) 34: 1435-1447, 2014.

16. Livak KJ and Schmittgen TD: Analysis of relative gene expression data using real-time quantitative PCR and the 2(-Delta Delta C(T)) Method. Methods 25: 402-408, 2001.

17. Gong M, Bi Y, Jiang W, Zhang Y, Chen L, Hou N, Liu Y, Wei X, Chen $\mathrm{J}$ and $\mathrm{Li} \mathrm{T}$ : Immortalized mesenchymal stem cells: An alternative to primary mesenchymal stem cells in neuronal differentiation and neuroregeneration associated studies. J Biomed Sci 18: 87, 2011.

18. Kishimoto T: Interleukin-6: Discovery of a pleiotropic cytokine. Arthritis Res Ther 8 (Suppl 2): S2, 2006.

19. Kishimoto T: IL-6: From its discovery to clinical applications. Int Immunol 22: 347-352, 2010.

20. Woodcock T and Morganti-Kossmann MC: The role of markers of inflammation in traumatic brain injury. Front Neurol 4: 18, 2013.

21. Arroyo DS, Soria JA, Gaviglio EA, Rodriguez-Galan MC and Iribarren P: Toll-like receptors are key players in neurodegeneration. Int Immunopharmacol 11: 1415-1421, 2011.

22. Wang YC, Lin S and Yang QW: Toll-like receptors in cerebral ischemic inflammatory injury. J Neuroinflammation 8: 134, 2011.

23. Vogel SN, Fitzgerald KA and Fenton MJ: TLRs: Differential adapter utilization by toll-like receptors mediates TLR-specific patterns of gene expression. Mol Interv 3: 466-477, 2003.

24. Marsh BJ, Williams-Karnesky RL and Stenzel-Poore MP: Toll-like receptor signaling in endogenous neuroprotection and stroke. Neuroscience 158: 1007-1020, 2009.

25. Crack PJ and Bray PJ: Toll-like receptors in the brain and their potential roles in neuropathology. Immunol Cell Biol 85: 476-480, 2007.

26. Mohamed MR and McFadden G: NFאB inhibitors: Strategies from poxviruses. Cell Cycle 8: 3125-3132, 2009.

27. Takeda $\mathrm{K}$ and Akira S: Toll-like receptors in innate immunity. Int Immunol 17: 1-14, 2005.

28. Akhmatova NK, Egorova NB, Kurbatova EA and Akhmatov EA: Activation of innate immunity by bacterial ligands of toll-like receptors. Front Immunol 5: 89, 2014. 